\title{
1 Rotavirus as an Expression Platform of the SARS-CoV-2 Spike Protein
}

3 Asha A. Philip and John T. Patton*

4 Department of Biology, Indiana University, Bloomington, IN 47405, USA

6 *Correspondence: John T. Patton, Department of Biology, Indiana University, 212 S.

7 Hawthorne Drive, Simon Hall 011, Bloomington, IN 47405 USA

9 Email addresses: Asha A. Philip, aaphilip@iu.edu; John T. Patton.jtpatton@iu.edu

11 Running Head: Rotavirus expressing SARS-CoV-2 spike

12

13 Word Counts: Abstract (250), Text (3318)

14 


\section{Abstract}

16 Rotavirus, a segmented double-stranded RNA virus, is a major cause of acute gastroenteritis in

17 young children. The introduction of live oral rotavirus vaccines has reduced the incidence of

18 rotavirus disease in many countries. To explore the possibility of establishing a combined

19 rotavirus-SARS-CoV-2 vaccine, we generated recombinant (r)SA11 rotaviruses with modified

20 segment 7 RNAs that contained coding sequences for NSP3 and FLAG-tagged portions of the

21 SARS-CoV-2 spike (S) protein. A 2A translational element was used to drive separate

22 expression of NSP3 and the S product. rSA11 viruses were recovered that encoded the S-protein

23 S1 fragment, N-terminal domain (NTD), receptor-binding domain (RBD), extended receptor-

24 binding domain (ExRBD), and S2 core (CR) domain (rSA11/NSP3-fS1, -fNTD, -fRBD, -

25 fExRBD, and -fCR, respectively). Generation of rSA11/fS1 required a foreign-sequence

26 insertion of 2.2-kbp, the largest such insertion yet made into the rotavirus genome. Based on

27 isopycnic centrifugation, rSA11 containing S sequences were denser than wildtype virus,

28 confirming the capacity of the rotavirus to accommodate larger genomes. Immunoblotting

29 showed that rSA11/-fNTD, -fRBD, -fExRBD, and -fCR viruses expressed S products of

30 expected size, with fExRBD expressed at highest levels. These rSA11 viruses were genetically

31 stable during serial passage. In contrast, rSA11/NSP3-fS1 failed to express its expected 80-kDa

32 fS1 product, for unexplained reasons. Moreover, rSA11/NSP3-fS1 was genetically unstable, with

33 variants lacking the S1 insertion appearing during serial passage. Nonetheless, these results

34 emphasize the potential usefulness of rotavirus vaccines as expression vectors of portions of the

35 SARS-CoV-2 S protein (e.g., NTD, RBD, ExRBD, and CR) with sizes smaller than the S1

36 fragment. 


\section{Importance}

38 Among the vaccines administered to children in the US and many other countries are those

39 targeting rotavirus, a segmented double-stranded RNA virus that is a major cause of severe

40 gastroenteritis. In this study, we have examined the feasibility of modifying the rotavirus genome

41 by reverse genetics, such that the virus could serve as an expression vector of the SARS-CoV-2

42 spike protein. Results were obtained showing that recombinant rotaviruses can be generated that

43 express domains of the SARS CoV-2 spike protein, including the receptor-binding domain

44 (RBD), a common target of neutralizing antibodies produced in individuals infected by the virus.

45 Our findings raise the possibility of creating a combined rotavirus-COVID-19 vaccine that could

46 be used in place of current rotavirus vaccines.

47

48 Key words. rotavirus, rotavirus vaccine, reverse genetics, Reoviridae, expression vector, SARS-

49 CoV-2, COVID-19 vaccine, spike protein 


\section{INTRODUCTION}

The impact of severe acute respiratory syndrome coronavirus 2 (SARS-CoV-2) on human

52 mortality and morbidity has stimulated broad ranging efforts to develop vaccines preventing

53 coronavirus disease 19 (COVID-19) (1,2). Given that the virus can cause asymptomatic and

54 symptomatic infections in individuals of all ages, including infants and young children,

55 comprehensive strategies to control the SARS-CoV-2 pandemic may require modification of

56 childhood immunization programs to include COVID-19 vaccines $(3,4)$. Among the vaccines

57 routinely administered to infants in the US and many other countries, are those targeting

58 rotavirus, a segmented double-stranded RNA (dsRNA) virus that is a primary cause of severe

59 acute gastroenteritis (AGE) in children during the first 5 years of life (5). The most widely used

60 rotavirus vaccines are given orally and formulated from live attenuated virus strains (6). These

61 vaccines induce the production of neutralizing $\operatorname{IgG}$ and $\operatorname{IgA}$ antibodies $(7,8,9)$ and have been

62 highly effective in reducing the incidence of rotavirus hospitalizations and mortality $(10,11)$.

Advances in rotavirus reverse genetics technologies have allowed the generation of

64 recombinant rotaviruses that serve as expression platforms of heterologous proteins (12-19). The

65 rotavirus genome consists of 11 segments of dsRNA, with a total size of $\sim 18.6 \mathrm{kbp}$ for group A

66 strains (rotavirus species A) typically associated with pediatric AGE (20). Most of the genome

67 segments contain a single open-reading frame (ORF); these encode the 6 structural (VP1-VP4,

68 VP6-VP7) or 6 nonstructural (NSP) viral proteins (21). The recently-developed rotavirus reverse

69 genetics systems consist of eleven T7 transcription (pT7) vectors, each directing synthesis of a

70 unique viral (+)RNA when transfected into baby-hamster kidney cells producing T7 RNA

71 polymerase (BHK-T7 cells). In some cases, support plasmids expressing capping enzymes

72 [African swine fever virus NP868R (22) or vaccinia virus D1L/D12R (18)] or fusion proteins 
73 [avian reovirus p10FAST (18)] are co-transfected with the pT7 vectors to enhance recovery of

74 recombinant viruses. Rotavirus reverse genetics systems have been used to mutate several of the

75 viral genome segments and to generate virus strains that express reporter proteins $(13,17,23-26)$.

76 Genome segment 7 of group A rotaviruses encodes NSP3 (36 kDa), an RNA-binding

77 protein that acts a translation enhancer of viral (+)RNAs and is expressed at moderate levels in

78 infected cells $(27,28)$. In a previous study, we showed that the single NSP3 ORF could be re-

79 engineered by reverse genetics to express two separate proteins through placement of a

80 teschovirus 2A translational stop-restart element at the end of the NSP3 ORF, followed by the

81 coding sequence for a heterologous protein (17). Through this approach, well-growing

82 genetically-stable recombinant rotaviruses have been generated that express NSP3 and one or

83 more fluorescent proteins (FPs) [e.g., mRuby (red), UnaG (green), TagBFP (blue), etc.] from

84 segment 7, an advance allowing study of rotavirus biology by live cell imaging (15). The NSP3

85 product of these recombinant viruses is functional, capable of dimerization and inducing the

86 nuclear accumulation of the cellular poly(A)-binding protein (16,17). Thus, recombinant

87 rotaviruses that express foreign proteins via addition of a $2 \mathrm{~A}$ element and coding sequence into

88 segment 7 downstream of the NSP3 ORF retain the full complement of functional viral ORFs.

As a step towards developing a combined rotavirus-SARS-CoV-2 vaccine, we explored

90 the possibility of generating recombinant rotaviruses that express regions of the SARS-CoV-2

91 spike (S) protein through re-engineering of the NSP3 ORF in segment 7. Trimers of the S protein

92 form crown-like projections that emanate from the lipid envelop surrounding the SARS-CoV-2

93 virion (29,30). Cleavage of the trimeric spikes by extracellular furin-like proteases generates S1

94 and S2 fragments, each which possess activities essential for virus entry (Fig. 1). The S1

95 fragment includes an N-terminal domain (NTD) and a receptor-binding domain (RBD), the latter 
96 mediating virus interaction with the cell surface receptor angiotensin-converting enzyme 2

97 (ACE2) (31). The S2 fragment is responsible for S-protein trimerization and contains fusion

98 domains that are essential for virus entry. SARS-CoV-2-specific antibodies with neutralizing

99 activity have been mapped to various regions of the S protein, including the NTD, RBD, and

100 fusion domains (32-35). We determined that by inserting $\mathrm{S}$ coding sequences into rotavirus

101 genome segment 7 downstream of the NSP3 ORF and a 2A element, well-growing genetically-

102 stable recombinant rotaviruses can be made that express all or portions of the S1 and S2

103 fragment. These findings raise the possibility of constructing rotavirus vaccine strains that are

104 not only capable of inducing immunological protective responses against rotavirus, but also

105 COVID-19.

106

107

\section{RESULTS AND DISCUSSION}

Modified segment 7 (NSP3) expression vectors containing SARS-CoV-2 S sequences.

109 To examine the possibility of using rotavirus as an expression platform for regions of the SARS-

110 CoV-2 S protein, we replaced the NSP3 ORF in the pT7/NSP3SA11 transcription vector with a

111 cassette comprised of the NSP3 ORF, a porcine teschovirus 2A element, and a coding sequence

112 of the S protein (Fig. 2). The cassette included a flexible GAG hinge between the coding

113 sequence for NSP3 and the 2A element and a 3x FLAG (f) tag between the coding sequences for

114 the $2 \mathrm{~A}$ element and the $\mathrm{S}$ region. This approach was used to generate a set of vectors

115 (collectively referred to as pT7/NSP3-CoV2/S vectors) that contained coding sequences for

116 SARS-CoV-2 S1 (pT7/NSP3-2A-fS1), NTD (pT7/NSP3-2A-fNTD), RBD (pT7/NSP3-2A-

117 fRBD), an extended form of the RBD (ExRBD) (pT7/NSP3-2A-fExRBD), and the S2 core

118 region (CR) including its fusion domains (pT7/NSP3-2A-fCR) (Fig. 1). The S sequences were 
119 inserted into the pT7/NSP3SA11 vector at the same site as used before in the production of

120 recombinant SA11 (rSA11) rotaviruses expressing FPs (15-17).

122 generate rSA11 viruses, BHK-T7 monolayers were transfected with a complete set of pT7/SA11

123 expression vectors, except pT7/NSP3SA11 was replaced with a pT7/NSP3-CoV2/S vector, and a

124 CMV expression plasmid (pCMV-NP868R) encoding the capping enzyme of African swine

125 fever virus. In transfection mixtures, plasmids encoding rotavirus NSP2 (pT7/NSP2SA11) and

126 NSP5 (pT7/NSP5SA11) were included at levels three-fold greater than the other pT7/SA11

127 vectors. BHK-T7 cells were overseeded with MA104 cells two days following transfection. The

128 BHK-T7/MA104 cell mixture was freeze-thawed three days later, and the rSA11 viruses were

129 recovered by plaque isolation and amplified by 1 or 2 cycles of growth in MA104 cells prior to

130 characterization (36). Properties of the rSA11 viruses are summarized in Table 1.

131 Based on gel electrophoresis, rSA11 viruses generated with pT7/NSP3-S vectors

132 (collectively referred to as rSA11/NSP3-CoV2/S viruses) contained segment 7 dsRNAs that

133 were much larger than that of wildtype rSA11 (rSA11/wt) virus (Fig. 3). Sequence analysis

134 confirmed that the segment 7 dsRNAs of the rSA11/NSP3-CoV2/S viruses matched the segment

1357 sequences present in the pT7/NSP3-CoV2/S vectors (data not shown). The re-engineered

136 segment 7 dsRNA of virus isolate rSA11/NSP3-fS1 had a length of $3.3 \mathrm{kbp}$, accounting for its

137 electrophoretic migration near the largest rotavirus genome segment (segment 1), which is

138 likewise $3.3 \mathrm{kbp}$ in length (Table 1, Fig. 3A). The segment 7 dsRNA of rSA11/NSP3-fS1

139 contains a 2.2-kbp foreign sequence insertion, the longest foreign sequence that has been

140 introduced into the segment $7 \mathrm{dsRNA}$, or for that matter, any rotavirus genome segment. The

141 previously longest 7 dsRNA engineered into rSA11 was the 2.4-kbp segment 7 dsRNA of 
142 rSA11/NSP3-fmRuby-P2A-fUnaG, which contained a cassette that encoded three proteins

143 (NSP3, UnaG, mRuby) (17). The total genome size of rSA11/NSP3-fS1 is $20.8 \mathrm{kbp}, 12 \%$ greater

144 than that of rSA11/wt (37). This is the largest genome known to exist within a rotavirus isolate

145 and demonstrates the capacity of rotavirus to replicate and package large amounts of foreign

146 sequence.

147 The segment 7 dsRNAs of virus isolates, rSA11/NSP3-fNTD, -fRBD, -fExRBD, and -

$148 \mathrm{fCR}$, were determined to have lengths of $2.1,1.8,2.1$, and $2.3 \mathrm{kbp}$, respectively (Table 1), and as

149 expected from their sizes, migrated on RNA gels between rotavirus genome segments 3 (2.6 kbp)

150 and 5 (1.6 kbp) (Fig. 3). The segment 7 dsRNAs of the rSA11/NSP3-fNTD, -fRBD, -fExRBD,

151 and -fCR isolates contained foreign sequence insertions of 1.0, 0.7, 1.0, and $1.2 \mathrm{kbp}$,

152 respectively, significantly smaller that the $2.1 \mathrm{kBP}$ foreign sequence insertion of rSA11/NSP3-

153 fS1. The smaller sizes of the foreign-sequence insert in the segment 7 RNAs of rSA11/NSP3-

154 fNTD, -fRBD, -fExRBD, and -fCR provide additional genetic space that can be used to add

155 routing and localization signals to $\mathrm{S}$ protein products, which may enhance their antigen

156 processing and presentation, recognition by T cells, and trafficking to immune cells. For

157 example, the extra genetic space can be used to add an N-terminal ER trafficking signal and a C-

158 terminal plasma-membrane localization signal to the ExRBD, along with internal coiled-coil

159 cassettes, that may favor surface presentation of a multimerized form of the ExRBD capable of

160 inducing enhanced production of SARS-CoV-2 neutralizing antibodies.

161 Consistent with previous studies examining the phenotypes of rSA11 isolates expressing

162 FPs (16-17), the sizes of plaques formed by rSA11/NSP3-CoV2/S viruses were smaller than

163 plaques formed by rSA11/wt. Similarly, rSA11 viruses containing S-protein coding sequences

164 grew to maximum titers that were up to $0.5-1 \log$ lower than $\mathrm{rSA} 11 / \mathrm{wt}$. The reason for the 
smaller plaques and lower titers of the rSA11/NSP3-CoV2/S viruses is unknown, but may reflect

167 segment 7 dsRNAs during viral replication. Alternatively, it may reflect the longer time required

168 to translate segment $7(+)$ RNAs that contain S-protein coding sequences.

$170 \mathrm{rSA} 11 / \mathrm{NSP} 3-\mathrm{CoV} 2 / \mathrm{S}$ viruses expressed products from their S sequences, lysates prepared from

171 MA104 cells infected with these viruses were examined by immunoblot assay using FLAG- and

172 RBD-specific antibodies (Fig. 4A, B). Immunoblots probed with FLAG antibody showed that

173 rSA11/NSP3-fNTD, -fExRBD, -fRBD, and -fCR viruses generated S products and that their

174 sizes were as predicted for an active 2A element in the segment 7 ORF: fNTD (34.8 kDa),

175 fExRBD (35.2 kDa), fRBD (24.3 kDa), and fCR (42.9 kDa) (Table 1). Immunoblot assays

176 indicated that the rSA11/NSP3-fExRBD yielded higher levels of S product than any of the other

$177 \mathrm{rSA} 11 / \mathrm{NSP} 3-\mathrm{CoV} 2 / \mathrm{S}$ viruses. The basis for the higher levels of the fExRBD product is unclear,

178 but does not correlate with increased levels of expression of other viral products, such as NSP3

179 and VP6. Nonetheless, the high levels of ExRBD expression by rSA11/NSP3-fExRBD suggests

180 that such viruses may be best suited in pursing the development of combined rotavirus/COVID

181 vaccines.

FLAG antibody did not detect the expected 79.6-kDa fS1 product in cells infected with

183 rSA11/NSP3-fS1 (Fig. 4A). The S1 coding sequence in the segment 7 ORF includes an N-

184 terminal signal sequence which, in SARS-CoV-2 infected cells, is cleaved from the S1 protein

185 during synthesis on the endoplasmic reticulum (ER) $(29,38)$. Cleavage of the signal sequence

186 may have removed the upstream 3x FLAG tag from a S1 product, preventing its detection by the

187 FLAG antibody. It is also possible that glycosylation and/or degradation of the $79.6 \mathrm{kDa}-\mathrm{S} 1$ 
188 product by ER-associated proteases may have prevented the protein's detection. In addition,

189 rotavirus which usurps and possibly remodels the ER in support of glycoprotein (NSP4 and VP7)

190 synthesis and virus morphogenesis may perturb ER-interaction with the $\mathrm{S}$ signal sequence in

191 such a way to prevent S1 synthesis (21). Interestingly, all the rSA11/NSP3-CoV2/S viruses,

192 including rSA11/NSP3-fS1, generated 2A read-through products that were detectable using

193 FLAG antibody. Thus, the 2A stop-start element in the rSA11/NSP3-2A-CoV2/S viruses was not

194 fully active, which is consistent with previous reports analyzing the functionality of 2A elements

195 within cells (39-41). However, with the exception of the rSA11/NSP3-fS1, all the viruses

196 generated more 2A-cleaved S product than read-through product. Mutation of residues in and

197 around the 2A element, including the inclusion of flexible linker sequences, may decrease the

198 relative frequency of read through (42-43).

Lysates from MA104 cells infected with rSA11/wt, rSA11/NSP3-fRBD, and

200 rSA11/NSP3-fExRBD were also probed with a RBD-specific polyclonal antibody prepared

201 against a peptide mapping to the C-terminal end of the RBD domain (ProSci 9087). The RBD

202 antibody recognized the fExRBD product of the rSA11/NSP3-fExRBD virus, but not the fRBD

203 product of rSA11/NSP3-fRBD (Fig. 4B), presumably because the latter product lacked the

204 peptide sequence used in generating the ProSci RBD antibody. To gain insight into whether the

205 fRBD and fExRBD products folded into native structures mimicking those present in the SARS-

206 CoV-2 S protein, lysates prepared from MA104 cells infected with rSA11/NSP3-fRBD and

207 rSA11/NSP3-fExRBD were probed by pulldown assay using an anti-RBD conformation-

208 dependent neutralizing monoclonal antibody (GeneTex CR3022). As shown in Fig. 4C, the

209 CR3022 immunoprecipitate included fExRBD, indicating that this product included a

210 neutralizing epitope found in authentic SARS-CoV-2 S protein. Thus, at least some of the RBD 
211 product of rSA11/NSP3-fExRBD has likely folded in a conformation capable of inducing a

212 protective antibody response. Unlike the successful pulldown of ExRBD with CR3022 antibody,

213 it was not clear if the antibody likewise immunoprecipitated the fRBD product of rSA11/NSP3-

214 fRBD. This uncertainty stems from the light chain of the CR3022 antibody obscuring the

215 electrophoretically closely-migrating fRBD product in immunoblot assays (Fig. 4C).

216 Expression of the ExRBD and RBD products by rSA11s during rotavirus infection.

217 To gain insight into fExRBD and fRBD expression during virus replication, MA104 cells were

218 infected with rSA11/wt, rSA11/NSP3-fExRBD or rSA11/NSP3-fRBD and then harvested at

219 intervals between 0 and $12 \mathrm{hr}$ p.i. Analysis of the infected cell lysates by immunoblot assay

220 showed that fExRBD and fRBD were readily detectable by 4 h p.i., paralleling the expression of

221 rotavirus proteins NSP3 and VP6 (Fig. 5). Increased levels of fExRBD and fRBD were present at

2228 and $12 \mathrm{~h}$ p.i., without obvious accumulation of FLAG-tagged products of smaller sizes. Thus,

223 the fExRBD and fRBD products appear to be relatively stable.

Density of rSA11 virus particles containing S sequences. The introduction of $S$

225 sequences into the rSA11/NSP3-CoV2/S viruses increased the size of their viral genomes by 1.0

226 to $2.5 \mathrm{kbp}$ beyond that of SA11/wt. Assuming the rSA11/NSP3-CoV2/S viruses are packaged

227 efficiently and contain a complete constellation of 11 genome segments, the increased content of

228 dsRNA within the core of rSA11/NSP3-CoV2/S particles should cause their densities to be

229 greater than that of SA11/wt particles. To explore this possibility, rSA11/wt (18.6-kbp genome),

$230 \mathrm{rSA} 11 / \mathrm{NSP} 3-\mathrm{fExRBD}(19.5 \mathrm{kbp})$ and rSA11/NSP3-fS1 (20.8 kbp) were amplified in MA104

231 cells. The infected-cell lysates were then treated with EDTA to convert rotavirus virions (triple-

232 layered particles) into double-layered particles (DLPs). The particles were centrifuged to

233 equilibrium on $\mathrm{CsCl}$ gradients (Fig. 6) and the density of the DLP bands determined by 
234 refractometry. The analysis indicated that the density of rSA11/NSP3-fExRBD DLPs (1.386

$235 \mathrm{~g} / \mathrm{cm}^{3}$ ) was greater than SA11/wt DLPs $\left(1.381 \mathrm{~g} / \mathrm{cm}^{3}\right)$ (panel A) and similarly, the density of

236 rSA11/NSP3-fS1 DLPs $\left(1.387 \mathrm{~g} / \mathrm{cm}^{3}\right)$ was greater that SA11/wt DLPs $\left(1.38 \mathrm{~g} / \mathrm{cm}^{3}\right)$ (panel B).

237 Analysis of the banded DLPs by gel electrophoresis confirmed that they contained the expected

238 constellation of eleven genome segments. To confirm that the density of rSA11/NSP3-fS1 DLPs

239 was different that rSA11/wt DLPs, infected-cell lysates containing each of these viruses were

240 pooled, treated with EDTA, and the viral DLPs in the combined sample banded by centrifugation

241 on a $\mathrm{CsCl}$ gradient (Fig. 6, panel E). Analysis of the gradient revealed the presence of two bands

242 of particles, indicating that rSA11/NSP3-fSA11-fS1 and rSA11/wt DLPs were of different

243 densities. Gel electrophoresis of the combined DLP bands showed, as expected, that both

244 rSA11/NSP3-fSA11-fS1 and rSA11/wt were present. Taken together, these results demonstrate

245 that rSA11/NSP3-CoV-2/S virions contain complete genome constellations despite the fact that

246 their genome sizes are significantly greater than that of wildtype SA11 virus. Indeed, the 20.8-

$247 \mathrm{kbp}$ rSA11/NSP3-fS1 genome is $12 \%$ greater in size than the 18.6-kbp rSA11/wt genome (Table

248 1). Thus, the rotavirus core has space to accommodate large amounts of additional foreign

249 sequence. How the dsRNA within the core is re-distributed to accommodate large amounts of

250 additional sequence is not known, but clearly the core remains a transcriptionally-active

251 nanomachine despite the additional sequence. Whether other genome segments can be

252 engineered similarly to segment 7 of rSA11/NSP3-fS 1 to include $2 \mathrm{~kb}$ of additional sequence

253 remains to be determined. The maximum packaging capacity of the core also remains to be

254 determined. 
MA104 cells infected with 1:1000 dilutions of cell lysates at each round. Electrophoretic analysis of the dsRNAs recovered from cells infected with rSA11/NSP3-fNTD, -fRBD, -ExRBD, or ExCR showed no changes in the sizes of any of the 11 genome segments over 5 rounds of

260 passage (P1-P5), including segment 7, indicating that these viruses were genetically stable. In

261 contrast, serial passage of rSA11/NSP3-S1 showed evidence of instability. By the third round of 262 passage, novel genome segments were appearing that were smaller than the 3.3-kbp segment 7

263 RNA. With continued passage, four novel segments (R-1 to R-4) became prominent and the 3.3-

264 kbp segment 7 RNA was no longer detectable, suggesting that the high-passage virus pools (P3-

265 P6) were populated by variants containing segment 7 RNAs derived from the 3.3-kb segment 7

266 RNA through internal sequence deletion. To evaluate this possibility, 8 variants were recovered

267 from the P6 virus pool by plaque isolation, 4 with a large (L) plaque phenotype and 4 with a

268 small (S) plaque phenotype. Electrophoretic analysis of the genomes of the variants showed that

269 none contained the 3.3-kbp segment 7 RNA. Instead, 6 variants (L1, L2, L3, L4, S2, and S4)

270 contained the R3 segment, and the other two variants contained either the R1 (S1) or R2 (R2)

271 segment. No variants were recovered that contained the novel R4 segment. Sequencing showed

272 that the R1, R2, and R3 segments were in fact derivatives of the 3.3-kbp segment 7 RNA. The

273 R1, R2, and R3 RNAs all retained the complete 5'- and 3'-UTRs and NSP3 ORF of segment 7,

274 but contained sequence deletions of $1.0(\mathrm{R} 1), 1.5(\mathrm{R} 2)$, or $1.8(\mathrm{R} 3) \mathrm{kbp}$ of S1 coding sequence.

275 The fact that 6 of the 8 variants isolated by plaque assay contained the R3 segment suggests that

276 variants with this RNA may have a growth advantage over variants with the R1, R2, or R4

277 RNAs. Although genetic instability gave rise to rSA11/NSP3-fS1 variants lacking portions of the

278 S1 ORF, none were identified that lacked portions of the NSP3 ORF. This suggests that NSP3

279 may be essential for virus replication, which would explain the failure of previous efforts by us 
to recover viable rSA11s encoding truncated forms of NSP3 through insertion of stop codons in the NSP3 ORF (data not shown).

Summary. We have shown that reverse genetics can be used to generate recombinant rotaviruses that express, as separate products, portions of the SARS-CoV-2 S protein, including its immunodominant RBD. These results indicate that it may be possible to develop rotaviruses as vaccine expression vectors, providing a path for generating oral live-attenuated rotavirusCOVID-19 combination vaccines able to induce immunological protective responses against both rotavirus and SARS-CoV-2. Such combination vaccines would be designed for use in infants and young children and would allow the widespread distribution and administration of COVID-19-targeted vaccines by piggy backing onto current rotavirus immunization programs

290 used in the USA and many other countries, both developed and developing. In addition, our

291 findings raise the possibility that through the use of rotavirus as vaccine expression platforms,

292 rotavirus-based combination vaccines could be made against other enteric viruses including 293 norovirus, astrovirus, and hepatitis E virus.

295 2.2-kbp of foreign sequence, which is sufficient to encode the SARS-CoV-2 S1 protein.

296 However, in our hands, rSA11s encoding S1 were not genetically stable and failed to express the 297 appropriate S1 product, for reasons that are uncertain but under further investigation. Rotaviruses 298 carrying large amounts of foreign sequence are characteristically genetically unstable (this study 299 and data not shown), but those with foreign sequences of <1.0-1.5-kbp are stable over 5-10 300 rounds of serial passage at low MOI and, thus, can be developed into vaccine candidates. The 301 coding capacity provided by $1.0-1.5-\mathrm{kbp}$ of extra sequence is sufficient to produce recombinant 302 rotaviruses that encode the SARS-CoV-2 NTD, RBD, or S2 core along with trafficking signals 
303 that can promote engagement of S products with antigen-presenting cells and naive B-

304 lymphocytes. Current work is underway to gain insight how successful rotaviruses expressing

305 SARS-CoV-2 products are in inducing neutralizing antibodies in immunized animals.

MATERIALS AND METHODS

Cell culture. Embryonic monkey kidney cells (MA104) were grown in medium 199

309 (M199) containing 5\% fetal bovine serum (FBS) and 1\% penicillin-streptomycin. Baby hamster

310 kidney cells expressing T7 RNA polymerase (BHK-T7) were provided by Dr. Ulla Buchholz,

311 Laboratory of Infectious Diseases, NIAID, NIH, and were propagated in Glasgow minimum

312 essential media (GMEM) containing 5\% heat-inactivated fetal bovine serum (FBS), 10\%

313 tryptone-peptide broth, $1 \%$ penicillin-streptomycin, $2 \%$ non-essential amino acids, and $1 \%$

314 glutamine (36). BHK-T7 cells were grown in medium supplemented with 2\% Geneticin

315 (Invitrogen) with every other passage.

Plasmid construction. Recombinant SA11 rotaviruses were prepared using the plasmids

317 pT7/VP1SA11, pT7/VP2SA11, pT7/VP3SA11, pT7/VP4SA11, pT7/VP6SA11, pT7/VP7SA11,

318 pT7/NSP1SA11, pT7/NSP2SA11, pT7/NSP3SA11, pT7/NSP4SA11, and pT7/NSP5SA11

319 [https://www.addgene.org/Takeshi_Kobayashi/] and pCMV-NP868R (16). The plasmid

320 pT7/NSP3-P2A-fUnaG was produced, as described elesewhere, by fusing a DNA fragment

321 containing the ORF for P2A-3xFL-UnaG to the 3'-end of the NSP3 ORF in pT7/NSP3SA11

322 (17). A plasmid (pTWIST/COVID19spike) containing a full-length cDNA of the SARS-CoV-2 S

323 gene (GenBank MN908947.3) was purchased from Twist Bioscience. The plasmids pT7/NSP3-

324 2A-fNTD, pT7/NSP3-2A-fExRBD, pT7/NSP3-2A-fRBD, pT7/NSP3-2A-fCR, and pT7/NSP3-

325 2A-S1 were made by replacing the UnaG ORF in pT7/NSP3-2A-fUnaG with ORFs for the NTD, 
ExRBD, RBD, CR, and S1 regions, respectively, of the SARS-CoV-2 S protein, by In-Fusion

327 cloning. DNA fragments containing NTD, ExRBD, RBD, CR, and S1 coding sequences were

328 amplified from pTWIST/COVID19spike using the primer pairs NTD_For and NTD_Rev,

329 ExRBD_For and ExRBD_Rev, RBD_For and RBD_Rev, CR_For and CR_Rev, and S1_For and

330 S1_Rev, respectively (Table 2). Transfection quality plasmids were prepared commercially

331 (www.plasmid.com) or using Qiagen plasmid purification kits. Primers were provided by and 332 sequences determined by EuroFins Scientific.

Recombinant viruses. The reverse genetics protocol used to generate recombinant

334 rotaviruses was described in detail previously $(16,44)$. To summarize, BHK-T7 cells were

335 transfected with SA11 pT7 plasmids and pCMV-NP868R using Mirus TransIT-LT1 transfection

336 reagent. Two days later, the transfected cells were overseeded with MA104 cells and the growth

337 medium (serum-free) adjusted to a final concentration of $0.5 \mu \mathrm{g} / \mathrm{ml}$ trypsin. Three days later, the

338 BHK-T7/MA104 cell mixture was freeze-thawed 3-times and the lysates clarified by low-speed

339 centrifugation. Recombinant virus in clarified lysates were amplified by one or two rounds of

340 passage in MA104 cells maintained in serum-free medium containing $0.5 \mu \mathrm{g} / \mathrm{ml}$ trypsin.

341 Individual virus isolates were obtained by plaque purification and typically amplified 1 or 2

342 rounds in MA104 cells prior to analysis. Viral dsRNAs were recovered from infected-cell lysates

343 by Trizol extraction, resolved by electrophoresis on Novex $8 \%$ polyacrylamide gels (Invitrogen)

344 in Tris-glycine buffer, and detected by staining with ethidium bromide. Viral dsRNAs in gels

345 were visualized using a BioRad ChemiDoc MP Imaging System. The genetic stability of plaque

346 isolated rSA11s was assessed by serial passage as described previously (17).

347 Immunoblot analysis. MA104 cells were mock infected or infected with 5 PFU of

348 recombinant virus per cell and harvested at $8 \mathrm{~h}$ p.i. Cells were washed with cold phosphate- 
349 buffered saline (PBS), pelleted by low-speed centrifugation, and lysed by resuspending in lysis

350 buffer [300 mM NaCl, $100 \mathrm{mM}$ Tris-HCl, $\mathrm{pH}$ 7.4, 2\% Triton X-100, and 1x EDTA-free protease

351 inhibitor cocktail (Roche cOmplete)]. For immunoblot assays, lysates were resolved by

352 electrophoresis on Novex linear 8-16\% polyacrylamide gels and transferred to nitrocellulose

353 membranes. After blocking with phosphate-buffered saline containing 5\% non-fat dry milk, blots

354 were probed with guinea pig polyclonal NSP3 (Lot 55068, 1:2000) or VP6 (Lot 53963, 1:2000)

355 antisera (2), mouse monoclonal FLAG M2 (Sigma F1804, 1:2000), rabbit monoclonal PCNA

356 [13110S, Cell Signaling Technology (CST), 1:1000] antibody or rabbit anti-RBD (ProSci 9087;

357 1:200) antibody. Primary antibodies were detected using 1:10,000 dilutions of horseradish

358 peroxidase (HRP)-conjugated secondary antibodies: horse anti-mouse IgG (CST), anti-guinea

359 pig IgG (KPL), or goat anti-rabbit IgG (CST). Signals were developed using Clarity Western

360 ECL Substrate (Bio-Rad) and detected using a Bio-Rad ChemiDoc imaging system.

361 Immunoprecipitation assay. Mock-infected and infected cell lysates were prepared as

362 above. Lysates were mixed with a SARS-CoV-2 S1 specific monoclonal antibody (GeneTex

363 CR3022, 1:150 dilution) or an NSP2 monoclonal antibody (\#171, 1:200). After incubation at 4C

364 with gentle rocking for $18 \mathrm{~h}$, antigen-antibody complexes were recovered using Pierce magnetic

365 IgA/IgG beads (ThermoFisher Scientific), resolved by gel electrophoresis, and blotted onto

366 nitrocellulose membranes. Blots were probed with FLAG antibody (1:2000) to detect fRBD and

367 fExRBD and NSP2 antibody (1:2000).

CsCI gradient centrifugation. MA104-cell monolayers in 10-cm cell culture plates were infected with rSA11 viruses at an MOI of 5 and harvested at $12 \mathrm{~h}$ p.i. Cells were lysed by

370 adjusting media to $0.5 \%$ Triton X100 (Sigma) and incubation on ice for $5 \mathrm{~min}$. Lysates were then

371 clarified by centrifugation at $500 \mathrm{x} g$ at $4 \mathrm{C}$ for $6 \mathrm{~min}$. The clarified lysates were adjusted to 10 
$372 \mathrm{mM}$ EDTA and incubated for $1 \mathrm{~h}$ at $37 \mathrm{C}$ to cause the conversion of rotavirus TLPs to DLPs (36).

$373 \mathrm{CsCl}$ was added to samples to a density of $1.367 \mathrm{~g} / \mathrm{cm}^{3}$ and samples were centrifuged at 110,000

$374 \mathrm{x} g$ with a Beckman SW55Ti rotor at 8C for $22 \mathrm{~h}$. Fractions containing viral bands were

375 recovered using a micropipettor and fraction densities were determined using a refractometer.

376 Genetic stability of rSA11 viruses. Viruses were serially passaged on MA104-cell

377 monolayers using 1:1000 dilutions of infected cell lysates prepared in serum-free M199 medium

378 and $0.5 \mu \mathrm{g} / \mathrm{ml}$ trypsin. When cytopathic effects reached completion (4-5 days), cells were freeze-

379 thawed twice in their medium, and lysates were clarified by low-speed centrifugation. To recover

380 dsRNA, clarified lysates (600 ul) were extracted with Trizol (ThermoFisher Scientific). The

381 RNA samples were resolved by electrophoresis on $8 \%$ polyacrylamide gels and the bands of

382 dsRNA detected by ethidium-bromide staining.

383 GenBank accession numbers. Segment 7 sequences in rSA11 viruses have been

384 deposited in Genbank: wt (LC178572), NSP3-P2A-fNTD (MW059024), NSP3-P2A-fRBD

385 (MT655947), NSP3-P2A-ExRBD (MT655946), NSP3-P2A-fCR (MW059025), NSP3-P2A-S1

386 (MW059026), NSP3-P2A-S1/R1 (MW353715), NSP3-P2A-S1/R2 (MW353716), and NSP3-

387 P2A-S1/R3 (MW353717). See also Table 1.

\section{ACKNOWLEDGEMENT}

Our thanks go to lab members for their support and encouragement on this project. This

391 work was funded by National Institutes of Health grant R21AI144881, Indiana University Start-

392 Up Funding, and the Lawrence M. Blatt Endowment. 


\section{FIGURE LEGENDS}

394 Figure 1. Domains of the SARS-CoV-2 S protein expressed by rSA11. (A) S protein trimers

395 are cleaved at the S1/S2 junction by furin proconvertase and at the S2' site by the TMPRSS2

396 serine protease. The S1 fragment contains a signal sequence (SS), N-terminal domain (NTD),

397 receptor binding domain (RBD), and receptor binding motif (RBM). The S2 fragment contains a

398 trimeric core region, transmembrane anchor (TM), and fusion domain. (B) Portions of the S

399 protein expressed by recombinant rotaviruses are indicated. (C) Ribbon representations of the

400 closed conformation of the trimeric S protein (PDB 6VXX) showing locations of the RBD

401 (magenta), extended RBD (ExRBD, cyan), NTD (blue), core (CR, gold) domains and the S1

402 cleavage product (green).

403 Figure 2. Plasmids with modified segment 7 (NSP3) cDNAs used to generate rSA11 viruses

404 expressing regions of the SARS-CoV-2 S protein. Illustration indicates nucleotide positions of

405 the coding sequences for NSP3, porcine teschovirus 2A element, 3xFLAG (FL), and the

406 complete S1 or portions of the S1 (NTD, ExRBD, and RBD) and S2 (CR) proteins. The red

407 arrow notes the position of the 2A translational stop-restart site, and the asterisk notes the end of

408 the ORF. Sizes (aa) of encoded NSP3 and S products are in parenthesis. T7 (T7 RNA

409 polymerase promoter sequence), Rz (Hepatitis D virus ribozyme), UTR (untranslated region).

410 Figure 3. Properties of rSA11/NSP3-CoV2/S viruses expressing regions of the SARS-CoV-2

411 S protein. (A and B) dsRNA was recovered from MA104 cells infected with plaque-purified

412 rSA11 isolates, resolved by gel electrophoresis, and detected by ethidium-bromide staining.

413 RNA segments of rSA11/wt are labeled 1 to 11. Sizes (kbp) of segment 7 RNAs (black arrows)

414 of rSA11 isolates are indicated. Double-stranded RNA of rSA11/NSP3-fS1 serially passaged

415 twice (P1 and P2) in MA104 cells is shown in (A). (C) Plaque assays were performed using 
416 MA104 cells and detected by crystal-violet staining. (D) Titers reached by rSA11 isolates were

417 determined by plaque assay. Bars indicate standard deviations calculated from three separate

418 determinations.

419 Figure 4. Expression of SARS-CoV-2 S products by rSA11 viruses. (A, B) Whole cell lysates

420 (WCL) were prepared from cells infected with rSA11 viruses and examined by immunoblot

421 assay using (A) FLAG antibody to detect S products (NTD, ExRBD, RBD, CR, S1, and 2A read-

422 through products) and antibodies specific for rotavirus NSP3 and VP6 and cellular PCNA. Red

423 asterisks identify $2 \mathrm{~A}$ read-through products and blue asterisks identify $2 \mathrm{~A}$ cleavage products. (B)

424 Lysates prepared from MA104 cells infected with rSA11wt, rSA11/NSP3-fRBD and

425 rSA11/NSP3-fExRBD were examined by immunoblot assay using antibodies specific for RBD

426 (ProSci 9087), rotavirus VP6, and PCNA. (C) Lysates prepared from MA104 cells infected with

427 rSA11/wt, rSA11/NSP3-fRBD and rSA11/NSP3-fExRBD viruses were examined by

428 immunoprecipitation assay using a SARS-CoV-2 S1 specific monoclonal antibody (GeneTex

429 CR3022). Lysates were also analyzed with a NSP2-specific polyclonal antibody. Antigen-

430 antibody complexes were recovered using IgA/G beads, resolved by gel electrophoresis, blotted

431 onto nitrocellulose membranes, and probed with FLAG (fRBD and fExRBD) and NSP2

432 antibody. Molecular weight markers are indicated (kDa). Red arrows indicate fRBD and

433 fExRBD. fRBD comigrates near the Ig light chain $(\mathrm{Ig} / \mathrm{L})$. Ig heavy chain, $\mathrm{Ig} / \mathrm{H})$.

434 Figure 5. Production of RBD and ExRBD by rSA11 viruses during infection. MA104 cells

435 were mock infected or infected with rSA11/wt, rSA11/NSP3-fRBD, or rSA11/NSP3-fExRBD

436 (MOI of 5). Lysates were prepared from the cells at $0,4,8$, or $12 \mathrm{~h} \mathrm{p.i.} \mathrm{and} \mathrm{analyzed} \mathrm{by}$

437 immunoblot assay using antibodies specific for FLAG, NSP3, VP6, and PCNA. Red asterisks

438 identify 2A read-through products. Positions of molecular weight markers are indicated (kDa). 
Figure 6. Impact of genome size on rotavirus particle density. MA104 cells were infected with rSA11/wt, rSA11/NSP3-fExRBD, or rSA11/NSP3-fS1 viruses at an MOI of 5. At 12 h p.i.,

441 the cells were recovered, lysed by treatment with non-ionic detergent, and treated with EDTA to

442 convert rotavirus virions into DLPs. (A, B) DLPs were banded by centrifugation in CsCl

443 gradients and densities $\left(\mathrm{g} / \mathrm{cm}^{3}\right)$ were determined using a refractometer. (C) Lysates from

444 rSA11/wt and rSA11/NSP3-fS1 infected cells were combined and their DLP components banded 445 by centrifugation in a $\mathrm{CsCl}$ gradient. (D, E) Electrophoretic profile of the dsRNA genomes of

446 DLPs recovered from CsCl gradients. Panel D RNAs derive from DLPs in panel A and panel E

447 RNAs derive from DLPs in panel B and C. RNA segments of rSA11/wt are labeled 1 to 11.

448 Positions of segment 7 RNAs are indicated with red arrows.

449 Figure 7. Genetic stability of rSA11 strains expressing SARS-CoV-2 S domains. rSA11

450 strains were serially passaged 5 to 6 times (P1 to P5 or P6) in MA104 cells. (A) Genomic RNAs

451 were recovered from infected cell lysates and analyzed by gel electrophoresis. Positions of viral

452 genome segments are labeled. Position of modified segment 7 (NSP3) dsRNAs introduced into

453 rSA11 strains are denoted with black arrows. Genetic instability of the modified segment 7

454 (NSP3) dsRNA of rSA11/NSP3-fS1 yielded R1-R4 RNAs during serial passage. (B) Genomic

455 RNAs prepared from large (L1-L4) and small (S1-S4) plaque isolates of P6 rSA11/NSP3-fS1.

456 Segment 7 RNAs are identified as R1-R3, as in (A). (C) Organization of R1-R3 sequences

457 determined by sequencing of segment 7 RNAs of L1, S1, and S3 plaque isolates. Sequence

458 deletions are indicated with dashed lines. Regions of the S1 ORF that are no longer encoded by

459 the R1-R3 segment 7 RNAs are indicated by slashed green-white boxes. 


\section{References}

1. Dai L, Gao GF. 2021. Viral targets for vaccines against COVID-19. Nat Rev Immunol 21, 73-82.

2. Kaur SP, Gupta V. 2020. COVID-19 Vaccine: A comprehensive status report. Virus Research 288: 198114.

3. Ludvigsson JF. 2020. Systematic review of COVID-19 in children shows milder cases and a better prognosis than adults. Acta Paediatr 109(6):1088-1095.

4. Pollán M, Pérez-Gómez B, Pastor-Barriuso R, Oteo J, Hernán MA, Pérez-Olmeda M, Sanmartín JL, Fernández-García A, Cruz I, Fernández de Larrea N, Molina M, RodríguezCabrera F, Martín M, Merino-Amador P, León Paniagua J, Muñoz-Montalvo JF, Blanco F, Yotti R; ENE-COVID Study Group. 2020. Prevalence of SARS-CoV-2 in Spain (ENECOVID): a nationwide, population-based seroepidemiological study. Lancet 396 (10250):

5. Burke RM, Tate JE, Kirkwood CD, Steele AD, Parashar UD. 2019. Current and new rotavirus vaccines. Curr Opin Infect Dis 32: 435-444.

6. Folorunso OS, Sebolai OM. 2020. Overview of the development, impacts, and challenges of 477 live-attenuated oral rotavirus vaccines. Vaccines (Basel) 8(3): 341.

478 7. Ali A, Kazi AM, Cortese MM, Fleming JA, Moon S, Parashar UD, Jiang B, McNeal MM, 479 Steele D, Bhutta Z, Zaidi AK. 2015. Impact of withholding breastfeeding at the time of 480 vaccination on the immunogenicity of oral rotavirus vaccine--a randomized trial. PLoS One 10(6): e0127622. Erratum in: PLoS One 10(12): e0145568. 
8. Liu GF, Hille D, Kaplan SS, Goveia MG. 2017. Postdose 3 G1 serum neutralizing antibody as correlate of protection for pentavalent rotavirus vaccine. Hum Vaccin Immunother 13(10): 2357-2363.

9. Angel J, Steele AD, Franco MA. 2014. Correlates of protection for rotavirus vaccines: Possible alternative trial endpoints, opportunities, and challenges. Hum Vaccin Immunother

10. Leshem E, Tate JE, Steiner CA, Curns AT, Lopman BA, Parashar UD. 2015. Acute gastroenteritis hospitalizations among US children following implementation of the rotavirus vaccine. JAMA. 2015 313(22): 2282-4. Erratum in: JAMA 314(2): 188.

12. Kanai Y, Kawagishi T, Nouda R, Onishi M, Pannacha P, Nurdin JA, Nomura K, Matsuura, Sanderson C, Lamberti L, Levine M, Santosham M, Steele D. 2017. Estimating global, regional and national rotavirus deaths in children aged $<5$ years: Current approaches, new 
15. Philip AA, Herrin BE, Garcia ML, Abad AT, Katen SP, Patton JT. 2019. Collection of recombinant rotaviruses expressing fluorescent reporter proteins. Microbio Resour Announc 8(27): e00523-19.

16. Philip AA, Perry JL, Eaton HE, Shmulevitz M, Hyser JM, Patton JT. 2019. Generation of recombinant rotavirus expressing NSP3-UnaG fusion protein by a simplified reverse genetics system. J Virol 93: e01616-19.

17. Philip AA, Patton JT. 2020. Expression of separate heterologous proteins from the rotavirus NSP3 genome segment using a translational 2A stop-restart element. J Virol 94(18): e0095920.

18. Kanai Y, Komoto S, Kawagishi T, Nouda R, Nagasawa N, Onishi M, Matsuura Y, Taniguchi K, Kobayashi T. 2017. Entirely plasmid-based reverse genetics system for rotaviruses. Proc Natl Acad Sci USA 114: 2349-2354.

19. Komoto S, Fukuda S, Kugita M, Hatazawa R, Koyama C, Katayama K, Murata T, Taniguchi K. 2019. Generation of infectious recombinant human rotaviruses from just 11 cloned

20. Crawford SE, Ramani S, Tate JE, Parashar UD, Svensson L, Hagbom M, Franco MA, Greenberg HB, O'Ryan M, Kang G, Desselberger U, Estes MK. 2017. Rotavirus infection.

523 21. Trask SD, McDonald SM, Patton JT. 2012. Structural insights into the coupling of virion assembly and rotavirus replication. Nat Rev Microbiol 10: 165-177. 
by promoting reovirus protein expression, virion assembly, and RNA incorporation into

528 infectious virions. J Virol 91: e02416-16.

529 23. Criglar JM, Crawford SE, Zhao B, Smith HG, Stossi F, Estes MK. 2020. A genetically

530 engineered rotavirus NSP2 phosphorylation mutant impaired in viroplasm formation and

531 replication shows an early interaction between vNSP2 and cellular lipid droplets. J Virol

532 94(15): e00972-20.

533 24. Navarro, A., Trask, S.D., Patton, J.T. 2013. Generation of genetically stable recombinant

534 rotaviruses containing novel genome rearrangements and heterologous sequences by reverse

535 genetics. J Virol 87: 6211-6220.

536 25. Chang-Graham AL, Perry JL, Strtak AC, Ramachandran NK, Criglar JM, Philip AA, Patton

537 JT, Estes MK, Hyser JM. 2019. Rotavirus calcium dysregulation manifests as dynamic

538 calcium signaling in the cytoplasm and endoplasmic reticulum. Sci Rep 9(1):10822.

539 26. Komoto S, Kanai Y, Fukuda S, Kugita M, Kawagishi T, Ito N, Sugiyama M, Matsuura Y,

540 Kobayashi T, Taniguchi K. 2017. Reverse genetics system demonstrates that rotavirus

541 nonstructural protein NSP6 is not essential for viral replication in cell culture. J Virol 91:

$542 \quad \mathrm{e} 00695-17$.

543 27. Gratia M, Sarot E, Vende P, Charpilienne A, Baron CH, Duarte M, Pyronnet S, Poncet D.

544 2015. Rotavirus NSP3 is a translational surrogate of the poly(A)-binding protein-poly(A)

$545 \quad$ complex. J Virol 89: 8773-8782.

546 28. Piron M, Delaunay T, Grosclaude J, Poncet D. 1999. Identification of the RNA-binding,

547 dimerization, and eIF4GI-binding domains of rotavirus nonstructural protein NSP3. J Virol

$548 \quad 73: 5411-5421$. 
29. Duan L, Zheng Q, Zhang H, Niu Y, Lou Y, Wang H. 2020. The SARS-CoV-2 spike glycoprotein biosynthesis, structure, function, and antigenicity: implications for the design of spike-based vaccine immunogens. Front Immunol 11: 576622.

30. Huang Y, Yang C, Xu XF, Xu W, Liu SW. 2020. Structural and functional properties of SARS-CoV-2 spike protein: potential antivirus drug development for COVID-19. Acta Pharmacol Sin 41(9): 1141-1149.

31. Medina-Enríquez MM, Lopez-León S, Carlos-Escalante JA, Aponte-Torres Z, Cuapio A, Wegman-Ostrosky T. 2020. ACE2: the molecular doorway to SARS-CoV-2. Cell Biosci 10(1): 148 .

32. Rogers TF, Zhao F, Huang D, Beutler N, Burns A, He WT, Limbo O, Smith C, Song G, Woehl J, Yang L, Abbott RK, Callaghan S, Garcia E, Hurtado J, Parren M, Peng L, Ramirez

33. Graham C, Seow J, Huettner I, Khan H, Kouphou N, Acors S, Winstone H, Pickering S, Pedro Galao R, Jose Lista M, Jimenez-Guardeno JM, Laing AG, Wu Y, Joseph M, Muir L, Ng WM, Duyvesteyn HME, Zhao Y, Bowden TA, Shankar-Hari M, Rosa A, Cherepanov P, variant on neutralizing monoclonal antibodies recognizing diverse epitopes on SARS-CoV-2 Spike. bioRxiv [Preprint]. 2021 Feb 3:2021.02.03.429355.

34. Xiaojie S, Yu L, Lei Y, Guang Y, Min Q. 2021. Neutralizing antibodies targeting SARSCoV-2 spike protein. Stem cell research 50: 102125. 
35. Liu L, Wang P, Nair MS, Yu J, Rapp M, Wang Q, Luo Y, Chan JF, Sahi V, Figueroa A, Guo XV, Cerutti G, Bimela J, Gorman J, Zhou T, Chen Z, Yuen KY, Kwong PD, Sodroski JG, Yin MT, Sheng Z, Huang Y, Shapiro L, Ho DD. 2020. Potent neutralizing antibodies against multiple epitopes on SARS-CoV-2 spike. Nature 584(7821): 450-456.

36. Arnold M, Patton JT, McDonald SM. 2009. Culturing, storage, and quantification of rotaviruses. Curr Protoc Microbiol Chapter 15: Unit 15C.3.

37. Desselberger U. 2020. What are the limits of the packaging capacity for genomic RNA in the cores of rotaviruses and of other members of the Reoviridae? Virus Res. 276:197822.

38. Meyer B, Chiaravalli J, Gellenoncourt S, Brownridge P, Bryne DP, Daly LA, Walter M,

39. Roulston C, Luke GA, de Felipe P, Ruan L, Cope J, Nicholson J, Sukhodub A, Tilsner J, Ryan MD. 2016. '2A-like' signal sequences mediating translational recoding: a novel form of

40. Luke G, Escuin H, De Felipe P, Ryan M. 2010. 2A to the fore - research, technology and applications. Biotechnol Genet Eng Rev 26: 223-60. 
593 42. Sharma P, Yan F, Doronina VA, Escuin-Ordinas H, Ryan MD, Brown JD. 2012. 2A peptides

594 provide distinct solutions to driving stop-carry on translational recoding. Nucleic Acids Res.

$595 \quad 40(7): 3143-51$.

596 43. Shaimardanova AA, Kitaeva KV, Abdrakhmanova II, Chernov VM, Rutland CS, Rizvanov

597 AA, Chulpanova DS, Solovyeva VV. 2019. Production and application of multicistronic

598 constructs for various human disease therapies. Pharmaceutics 11(11): 580.

599 44. Philip AA, Dai J, Katen SP, Patton JT. 2020. Simplified reverse genetics method to recover

$600 \quad$ recombinant rotaviruses expressing reporter proteins. J Vis Exp 158: e61039.

601 45. Matthijnssens J, Ciarlet M, McDonald SM, Attoui H, Bányai K, Brister JR, Buesa J, Esona,

602 MD, Estes MK, Gentsch JR, Iturriza-Gómara M, Johne R, Kirkwood CD, Martella V,

603 Mertens PP, Nakagomi O, Parreño V, Rahman M, Ruggeri FM, Saif LJ, Santos N, Steyer A.,

604 Taniguchi K, Patton JT, Desselberger U, Van Ranst M. 2011. Uniformity of rotavirus strain

605 nomenclature proposed by the Rotavirus Classification Working Group (RCWG). Arch Virol

$606 \quad 156: 1397-1413$.

607 


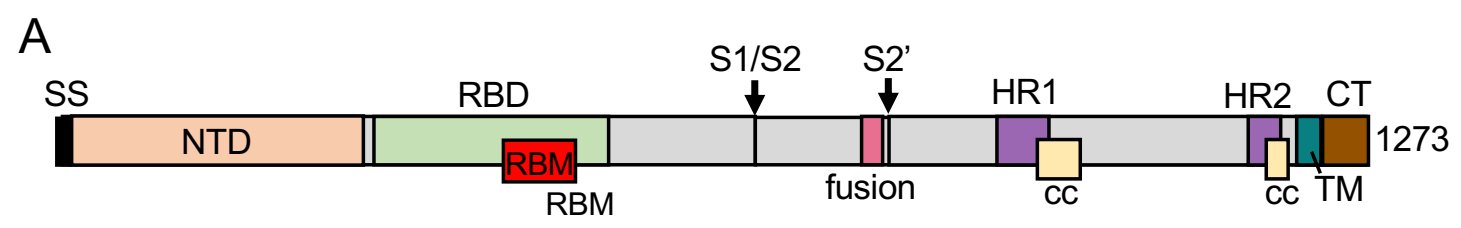

B

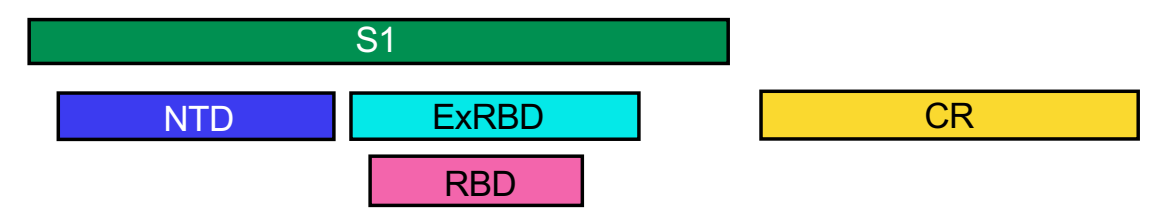

C
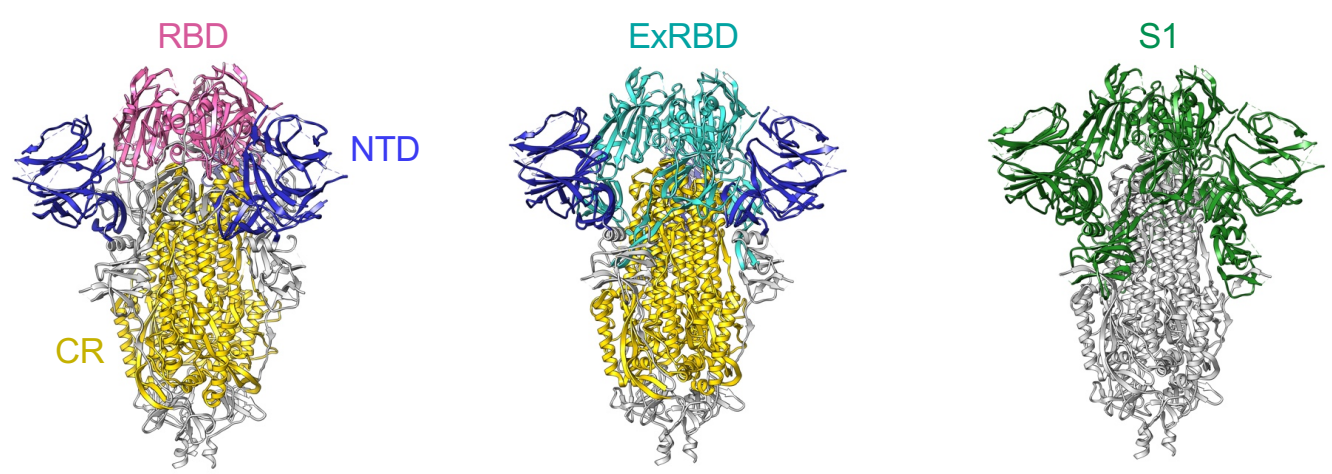

Figure 1. Domains of the SARS-CoV-2 S protein expressed by rSA11. (A) S protein trimers are cleaved at the S1/S2 junction by furin proconvertase and at the S2' site by the TMPRSS2 serine protease. The S1 fragment contains a signal sequence (SS), N-terminal domain (NTD), receptor binding domain (RBD), and receptor binding motif (RBM). The S2 fragment contains a trimeric core region, transmembrane anchor (TM), and fusion domain. (B) Portions of the S protein expressed by recombinant rotaviruses are indicated. (C) Ribbon representations of the closed conformation of the trimeric S protein (PDB 6VXX) showing locations of the RBD (magenta), extended RBD (ExRBD, cyan), NTD (blue), core (CR, gold) domains and the S1 cleavage product (green) . 


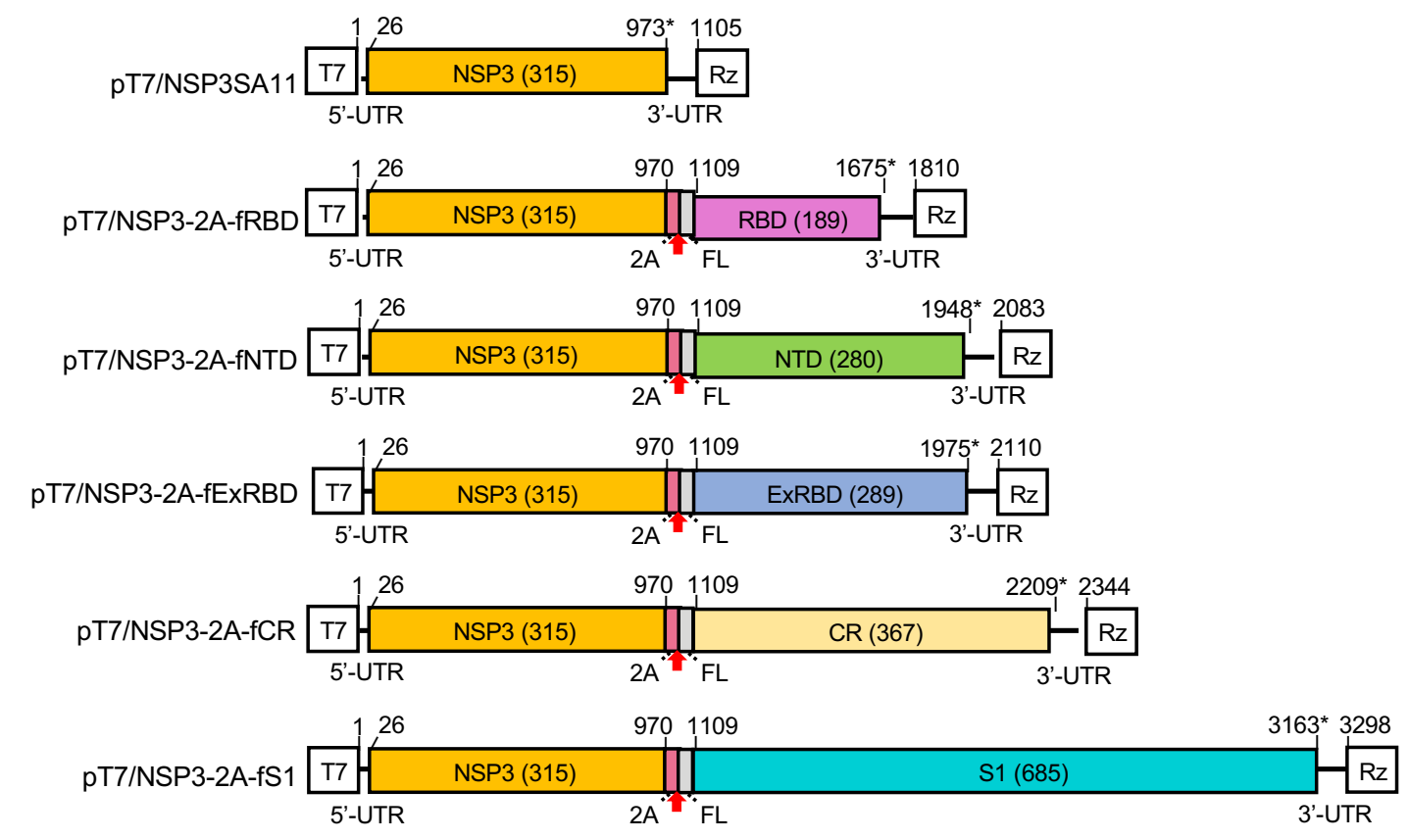

Figure 2. Plasmids with modified segment 7 (NSP3) cDNAs used to generate rSA11 viruses expressing regions of the SARS-CoV-2 S protein. Illustration indicates nucleotide positions of the coding sequences for NSP3, porcine teschovirus 2A element, $3 x$ FLAG (FL), and the complete S1 or portions of the S1 (NTD, ExRBD, and RBD) and S2 (CR) proteins. The red arrow notes the position of the 2A translational stop-restart site, and the asterisk notes the end of the ORF. Sizes (aa) of encoded NSP3 and S products are in parenthesis. T7 (T7 RNA polymerase promoter sequence), Rz (Hepatitis D virus ribozyme), UTR (untranslated region). 
A

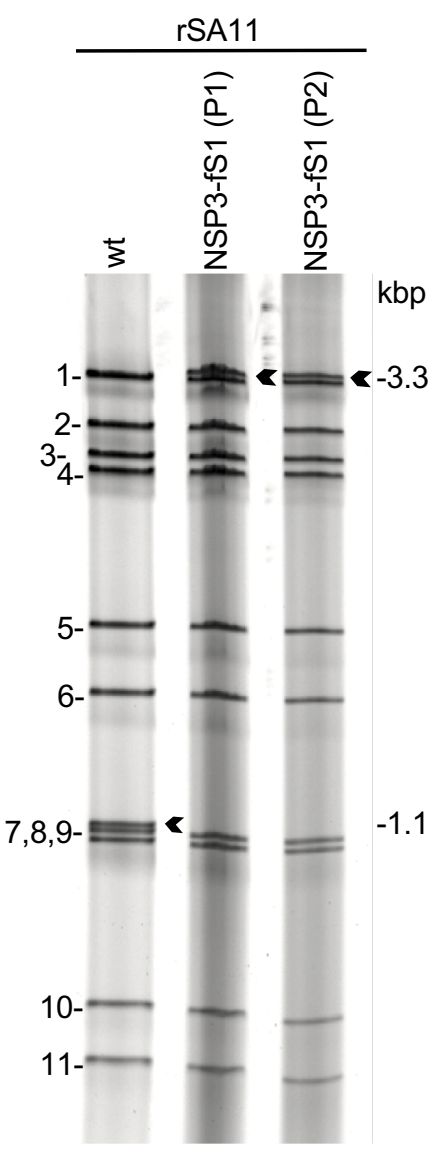

\section{C}

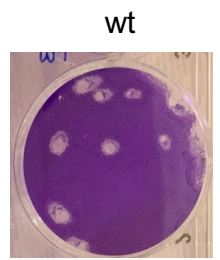

NSP3-fNTD

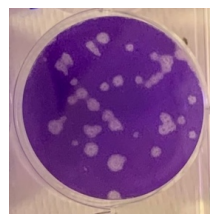

NSP3-fExRBD

NSP3-fCR
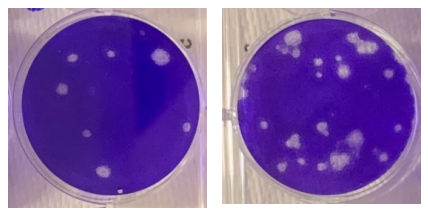

B

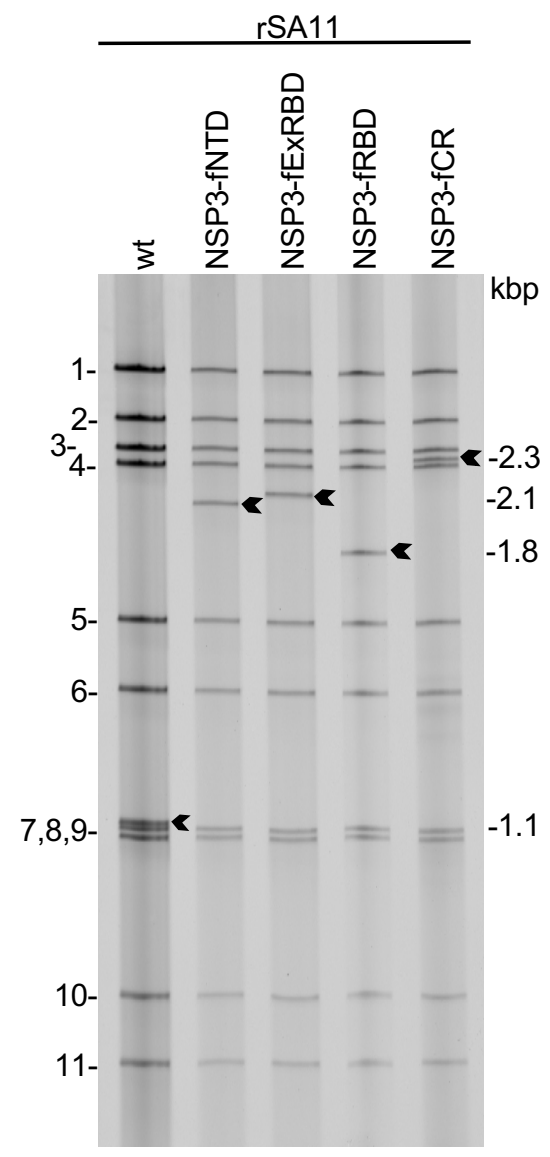

NSP3-fRBD

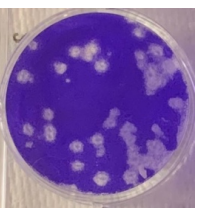

NSP3-fS1

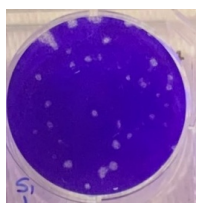

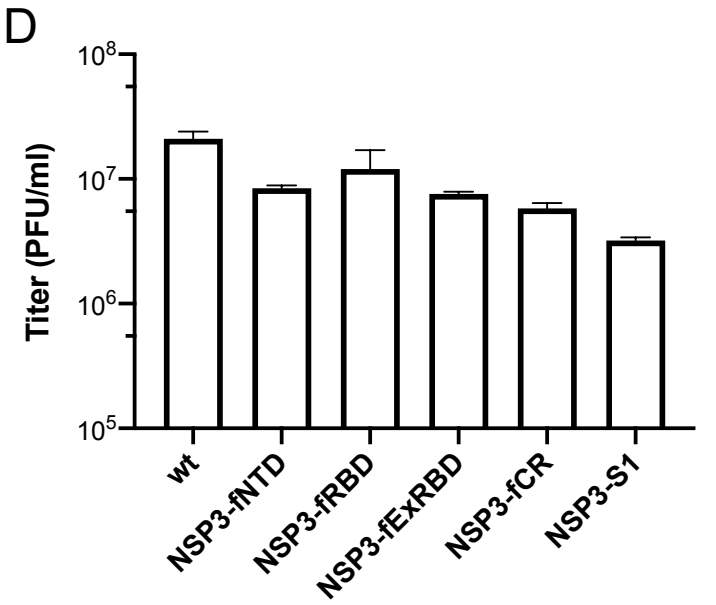

Figure 3. Properties of rSA11/NSP3-CoV2/S viruses expressing regions of the SARSCoV-2 S protein. (A and B) dsRNA was recovered from MA104 cells infected with plaquepurified rSA11 isolates, resolved by gel electrophoresis, and detected by ethidium-bromide staining. RNA segments of rSA11/wt are labeled 1 to 11. Sizes (kbp) of segment 7 RNAs (black arrows) of rSA11 isolates are indicated. Double-stranded RNA of rSA11/NSP3-fS1 serially passaged twice (P1 and P2) in MA104 cells is shown in (A). (C) Plaque assays were performed using MA104 cells and detected by crystal-violet staining. (D) Titers reached by rSA11 isolates were determined by plaque assay. Bars indicate standard deviations calculated from three separate determinations. 

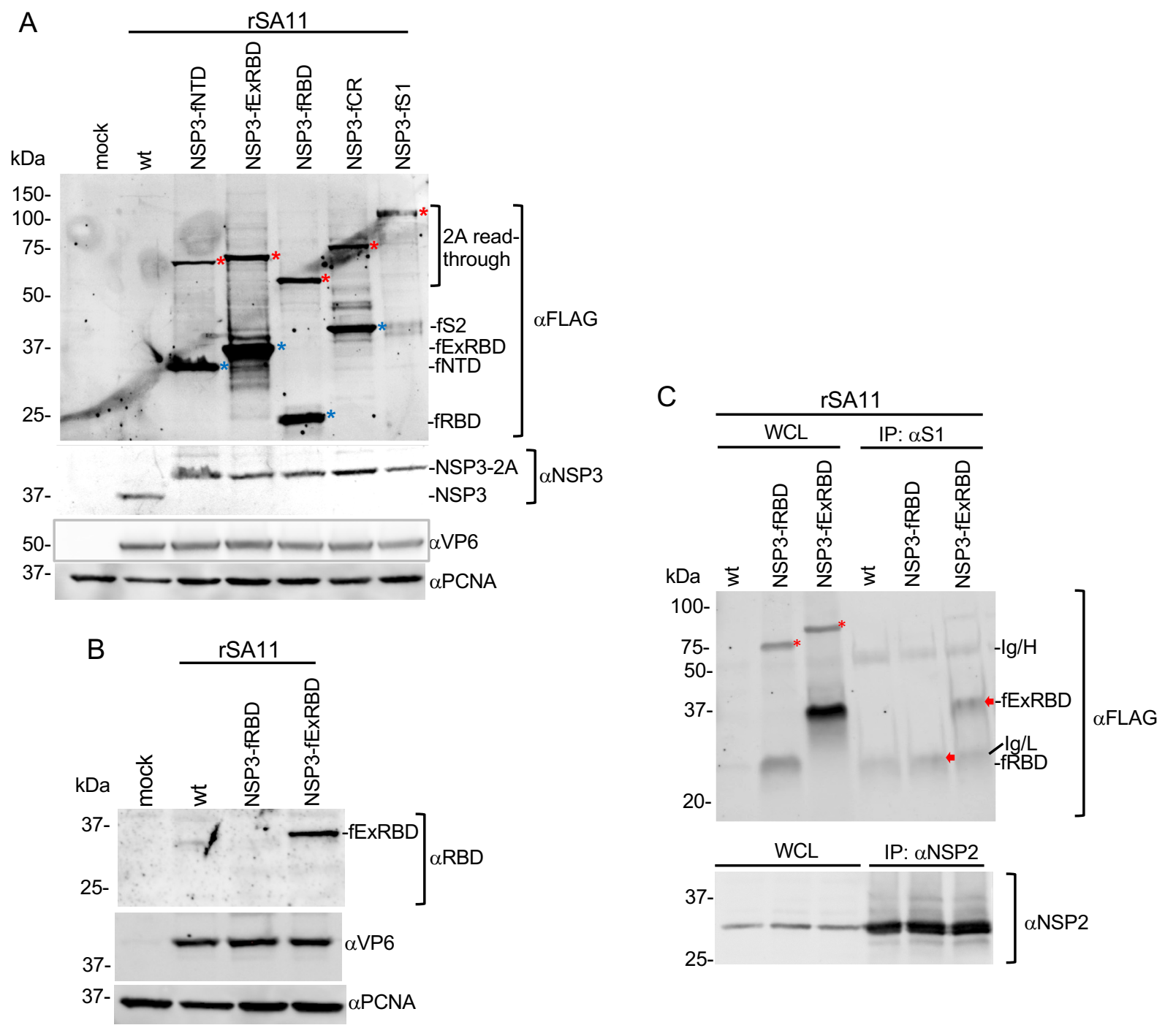

Figure 4. Expression of SARS-CoV-2 S products by rSA11 viruses. (A, B) Whole cell lysates (WCL) were prepared from cells infected with rSA11 viruses and examined by immunoblot assay using (A) FLAG antibody to detect S products (NTD, ExRBD, RBD, CR, S1, and 2A read-through products) and antibodies specific for rotavirus NSP3 and VP6 and cellular PCNA. Red asterisks identify 2A readthrough products and blue asterisks identify 2A cleavage products. (B) Lysates prepared from MA104 cells infected with rSA11wt, rSA11/NSP3-fRBD and rSA11/NSP3-fExRBD were examined by immunoblot assay using antibodies specific for RBD (ProSci 9087), rotavirus VP6, and PCNA. (C) Lysates prepared from MA104 cells infected with rSA11/wt, rSA11/NSP3-fRBD and rSA11/NSP3fExRBD viruses were examined by immunoprecipitation assay using a SARS-CoV-2 S1 specific monoclonal antibody (GeneTex CR3022). Lysates were also analyzed with a NSP2-specific polyclonal antibody. Antigen-antibody complexes were recovered using $\operatorname{IgA} / \mathrm{G}$ beads, resolved by gel electrophoresis, blotted onto nitrocellulose membranes, and probed with FLAG (fRBD and fExRBD) and NSP2 antibody. Molecular weight markers are indicated (kDa). Red arrows indicate fRBD and fExRBD. fRBD comigrates near the Ig light chain (Ig/L). Ig heavy chain, Ig/H). 


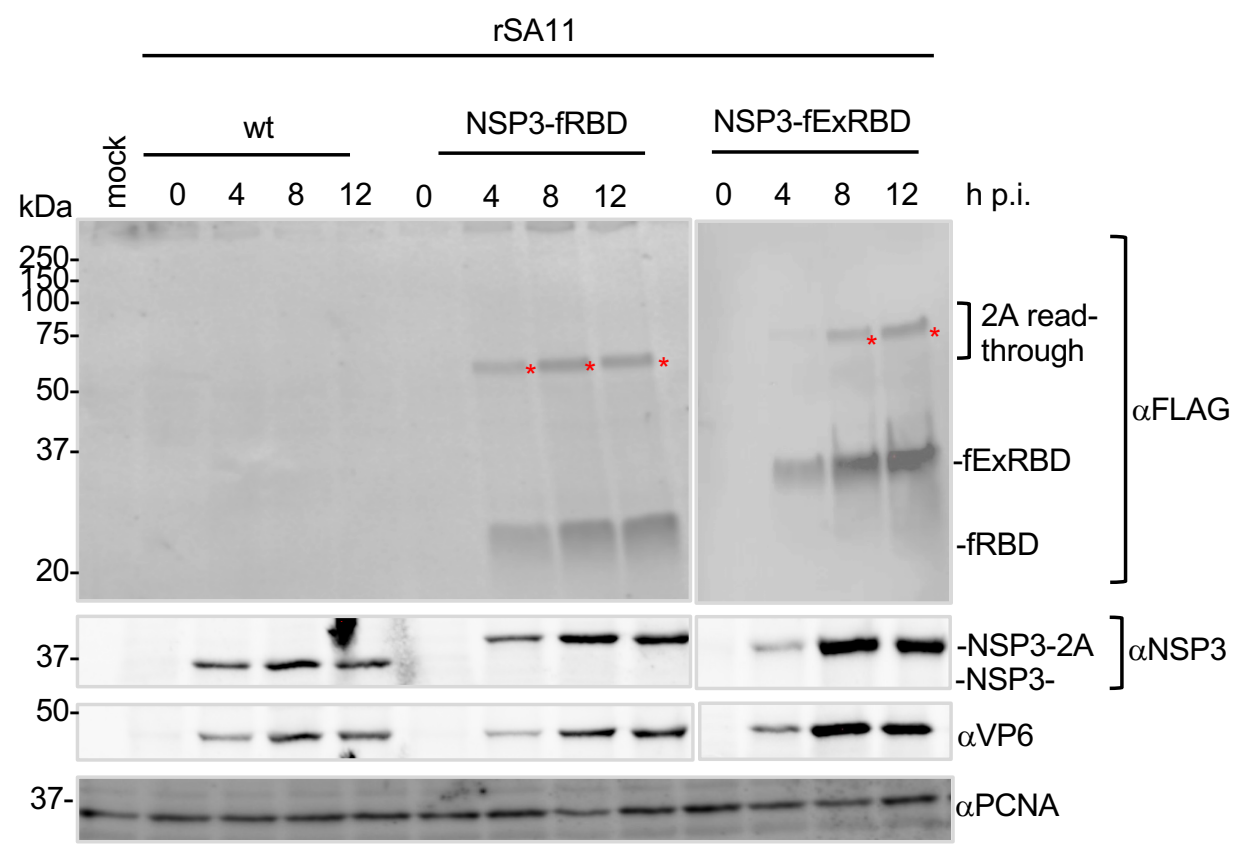

Figure 5. Production of RBD and ExRBD by rSA11 viruses during infection. MA104 cells were mock infected or infected with rSA11/wt, rSA11/NSP3-fRBD, or rSA11/NSP3-fExRBD (MOI of 5). Lysates were prepared from the cells at $0,4,8$, or $12 \mathrm{~h}$ p.i. and analyzed by immunoblot assay using antibodies specific for FLAG, NSP3, VP6, and PCNA. Red asterisks identify $2 \mathrm{~A}$ read-through products. Positions of molecular weight markers are indicated $(\mathrm{kDa})$. 


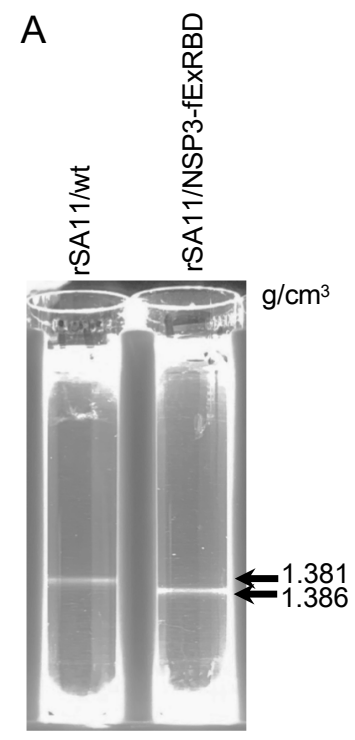

B

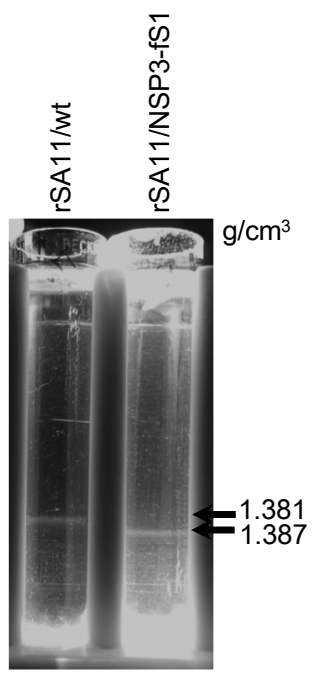

C

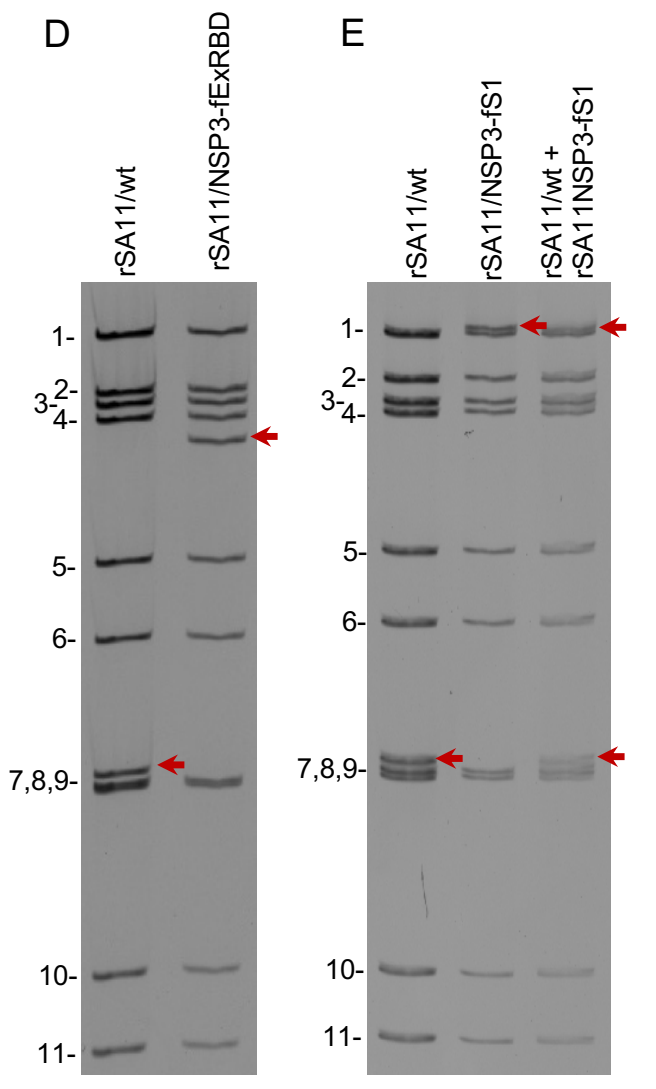

Figure 6. Impact of genome size on rotavirus particle density. MA104 cells were infected with rSA11/wt, rSA11/NSP3-fExRBD, or rSA11/NSP3-fS1 viruses at an MOI of 5. At $12 \mathrm{~h}$ p.i., the cells were recovered, lysed by treatment with non-ionic detergent, and treated with EDTA to convert rotavirus virions into DLPs. (A, B) DLPs were banded by centrifugation in $\mathrm{CsCl}$ gradients and their densities $\left(\mathrm{g} / \mathrm{cm}^{3}\right)$ determined using a refractometer. (C) Lysates from rSA11/wt and rSA11/NSP3-fS1 infected cells were combined and their DLP components banded by centrifugation in a CsCl gradient. (D,E) Electrophoretic profile of the dsRNA genomes of DLPs recovered from $\mathrm{CsCl}$ gradients. Panel D RNAs derive from DLPs in panel A and panel E RNAs derive from DLPs in panel B and C. RNA segments of rSA11/wt are labeled 1 to 11. Positions of segment 7 RNAs are indicated with red arrows. 

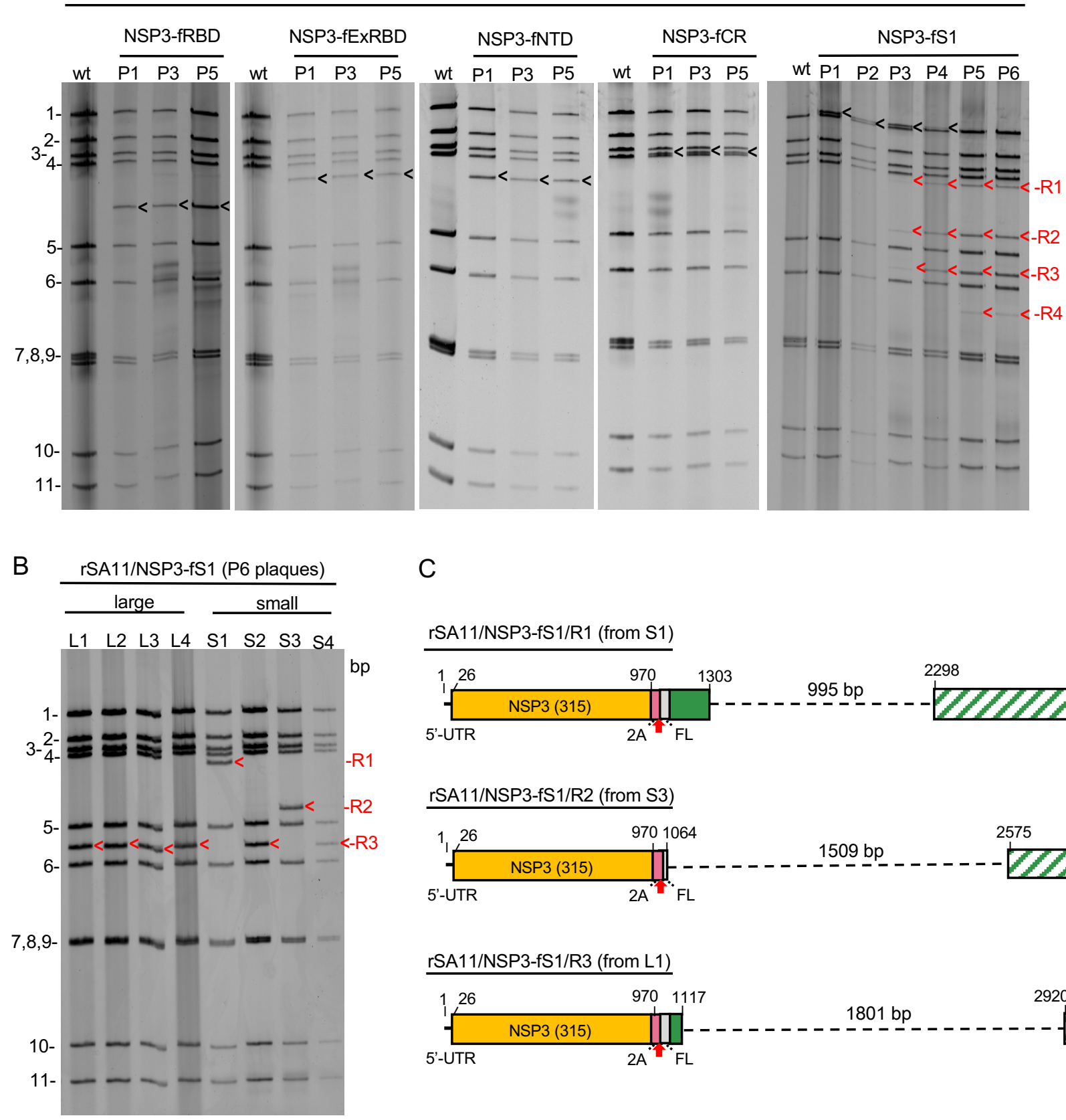

C

rSA11/NSP3-fS1/R1 (from S1)

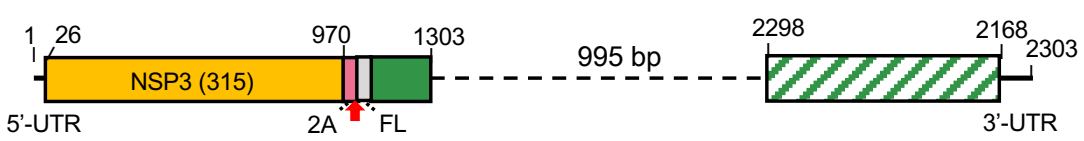

rSA11/NSP3-fS1/R2 (from S3)

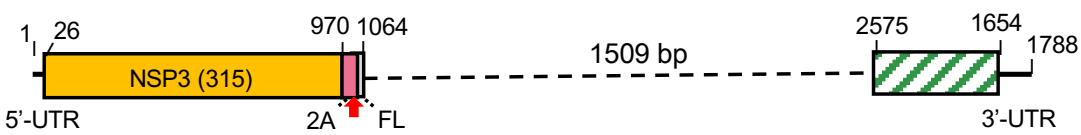

rSA11/NSP3-fS1/R3 (from L1)

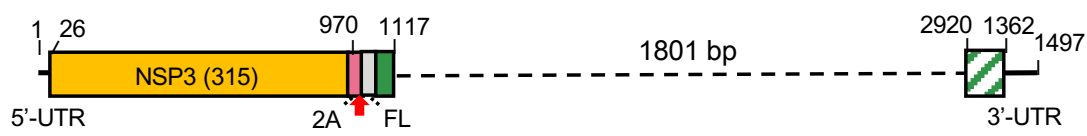

Figure 7. Genetic stability of rSA11 strains expressing SARS-CoV-2 S domains. rSA11 strains were serially passaged 5 to 6 times (P1 to P5 or P6) in MA104 cells. (A) Genomic RNAs were recovered from infected cell lysates and analyzed by gel electrophoresis. Positions of viral genome segments are labeled. Position of modified segment 7 (NSP3) dsRNAs introduced into rSA11 strains are denoted with black arrows. Genetic instability of the modified segment 7 (NSP3) dsRNA of rSA11/NSP3-fS1 yielded R1-R4 RNAs during serial passage. (B) Genomic RNAs prepared from large (L1-L4) and small (S1-S4) plaque isolates of P6 rSA11/NSP3-fS1. Segment 7 RNAs are identified as R1-R3, as in (A). (C) Organization of R1-R3 sequences determined by sequencing of segment 7 RNAs of L1, S1, and S3 plaque isolates. Sequence deletions are indicated with dashed lines. Regions of the S1 ORF that are no longer encoded by the R1-R3 segment 7 RNAs are indicated by slashed green-white boxes. 


\begin{tabular}{|c|c|c|c|c|c|c|c|c|}
\hline \multirow{2}{*}{\multicolumn{3}{|c|}{ Virus strain }} & \multicolumn{6}{|c|}{ Genome segment 7} \\
\hline & & & \multirow[b]{2}{*}{$\begin{array}{r}\text { RNA } \\
\text { (bp) }\end{array}$} & \multicolumn{4}{|c|}{ Protein product } & \multirow{2}{*}{$\begin{array}{c}\text { NCBI } \\
\text { accession } \\
\#\end{array}$} \\
\hline Abbreviated name & Formal name* & $\begin{array}{c}\text { Genome size/ } \\
\text { increase over wt } \\
\text { (bp) }\end{array}$ & & $\begin{array}{l}\text { uncleaved } \\
\text { (aa) }\end{array}$ & $\begin{array}{c}2 \mathrm{~A} \text { cleaved } \\
\text { (aa) }\end{array}$ & $\begin{array}{l}\text { uncleaved } \\
(\mathrm{kDa})\end{array}$ & $\begin{array}{c}2 \mathrm{~A} \text { cleaved } \\
(\mathrm{kDa})\end{array}$ & \\
\hline rSA11/wt & $\begin{array}{c}\text { RVA/Simian- } \\
\text { lab/USA/SA11wt/2019/G3P[2] }\end{array}$ & $18,559 / 0$ & 1105 & 315 & nd & 36.4 & nd & LC178572 \\
\hline rSA11/NSP3-fNTD & $\begin{array}{c}\text { RVA/Simian-lab/USA/SA11(NSP3- } \\
\text { P2A-CoV2:fNTD)/2020/G3P[2] }\end{array}$ & $19,537 / 978$ & 2083 & 641 & $336+305$ & 73.2 & $38.5+34.8$ & MW059024 \\
\hline rSA11/NSP3-fRBD & $\begin{array}{c}\text { RVA/Simian-lab/USA/SA11(NSP3- } \\
\text { P2A-CoV2:fRBD)/2020/G3P[2] }\end{array}$ & $19,264 / 705$ & 1810 & 550 & $336+214$ & 62.7 & $38.5+24.3$ & MT655947 \\
\hline rSA11/NSP3-fExRBD & $\begin{array}{l}\text { RVA/Simian-lab/USA/SA11(NSP3- } \\
\text { P2A-CoV2:fExRBD)/2020/G3P[2] }\end{array}$ & $19,564 / 1005$ & 2110 & 650 & $336+314$ & 74.7 & $38.5+35.2$ & MT655946 \\
\hline rSA11/NSP3-fCR & $\begin{array}{c}\text { RVA/Simian-lab/USA/SA11(NSP3- } \\
\text { P2A-CoV2:fCR)/2020/G3P[2] }\end{array}$ & $19,798 / 1239$ & 2344 & 728 & $336+392$ & 81.4 & $38.5+42.9$ & MW059025 \\
\hline rSA11/NSP3-fS1 & $\begin{array}{l}\text { RVA/Simian-lab/USA/SA11(NSP3- } \\
\text { P2A-CoV2:fS1)/2020/G3P[2] }\end{array}$ & $20,752 / 2193$ & 3298 & 1046 & $336+710$ & 118.1 & $38.5+79.6$ & MW059026 \\
\hline rSA11/NSP3-fS1/R1 & $\begin{array}{c}\text { RVA/Simian-lab/USA/SA11(NSP3- } \\
\text { P2A-CoV2:fS1/R1)/2020/G3P[2] }\end{array}$ & $19,757 / 1198$ & 2303 & 431 & $336+95$ & 49.6 & $38.5+11.1$ & MW353715 \\
\hline rSA11/NSP3-fS1/R2 & $\begin{array}{l}\text { RVA/Simian-lab/USA/SA11(NSP3- } \\
\text { P2A-CoV2:fS1/R2)/2020/G3P[2] }\end{array}$ & $19,233 / 683$ & 1789 & 367 & $336+31$ & 42.1 & $38.5+3.7$ & MW353716 \\
\hline rSA11/NSP3-fS1/R3 & $\begin{array}{c}\text { RVA/Simian-lab/USA/SA11(NSP3- } \\
\text { P2A-CoV2:fS1R3)/2020/G3P[2] }\end{array}$ & $18,951 / 392$ & 1497 & 410 & $336+74$ & 47.2 & $38.5+8.8$ & MW353717 \\
\hline
\end{tabular}

* Formal strain names were assigned according to Matthijnssens et al (45). nd: not determined, no 2A cleavage site present; wt: wild type 


\begin{tabular}{|c|c|}
\hline Primer & Sequence \\
\hline Vector_For & TGACCATTTTGATACATGTTGAACAATCAAATACAG \\
\hline Vector_Rev & GCTAGCCTTGTCATCGTCATCCT \\
\hline NTD_For & GATGACAAGGCTAGCTGTGTTAATCTTACAACCAGAACTCAATTACCCC \\
\hline NTD_Rev & GTATCAAAATGGTCAGTCAAGTGCACAGTCTACAGCATC \\
\hline ExRBD_For & GATGACAAGGCTAGCGGAATCTATCAAACTTCTAACTTTAGAGTCCAACCA \\
\hline ExRBD_Rev & GTATCAAAATGGTCATGTTATAACACTGACACCACCAAAAGAACA \\
\hline RBD_For & GATGACAAGGCTAGCTTGTGCCCTTTTGGTGAAGTTT \\
\hline RBD_Rev & GTATCAAAATGGTCAAGTTGCTGGTGCATGTAGAAGT \\
\hline CR_For & GATGACAAGGCTAGCTCTATTGCCATACCCACAAATTTTACTATTAGTGT \\
\hline CR_Rev & GTATCAAAATGGTCAAGTTGTGAAGTTCTTTTCTTGTGCAGG \\
\hline S1_For & GATGACAAGGCTAGCGTGTTTGTTTTTCTTGTTTTATTGCCACTAGTCT \\
\hline S1_Rev & GTATCAAAATGGTCAACGTGCCCGCCG \\
\hline
\end{tabular}

